\section{Il beneficio dei farmaci oncologici: commento all'articolo di Ballatori e colleghi}

\section{Dear Editor,}

Abbiamo letto con interesse l'articolo di Ballatori e colleghi (1). Si tratta di un contributo di attualità che, oltre ad arricchire la rivista, solleva una serie di ulteriori importanti questioni da approfondire (soprattutto nella prospettiva dei futuri percorsi italiani). Procediamo per punti:

Primo punto. Permane la tradizionale dualità di impostazioni [Overall Survival (OS) vs Progression Free Survival (PFS)] nella scelta del parametro che quantifica il beneficio incrementale. Come noto, questo parametro di "quantificazione" è definito, nel caso si scelga l'OS, come prolungamento della sopravvivenza sino a fine vita (unità di misura = tempo) oppure, nel caso si scelga il PFS, oltre che come prolungamento della sopravvivenza fino alla progressione, anche come miglioramento (assoluto o relativo che sia) della percentuale di pazienti event-free riferita a un dato tempo del follow-up (unità di misura = percentuale di pazienti event-free). In questa dualità di impostazioni merita, a nostro avviso, ricordare che il "primo modo" (miglioramento della OS) è molto più vantaggioso del secondo (miglioramento della PFS), soprattutto perché i benchmark economici già in uso da tanti anni (2-10) fanno riferimento al guadagno di sopravvivenza complessiva (es. costo per anno di vita guadagnato) e non alla progressione (a prescindere che quest'ultima sia espressa come tempo guadagnato oppure come percentuale eventfree migliorata). II "primo modo" va inoltre privilegiato perché tende a essere patologia-indipendente.

Secondo punto. Il metodo di Ballatori e colleghi, qualora misuri il beneficio in termini di OS, può essere applicato anche incorporando una soglia di riferimento, cioè un valore che esprima la willingness to pay (WTP) (misurata come costo per anno di vita guadagnato). Lo stesso non si può dire, quantomeno al momento attuale, qualora si misuri il beneficio in termini di PFS, per mancanza di soglie di accettabilità per la PFS. Forse è per tale motivo che gli esempi descritti nell'articolo sono tutti impostati per eseguire il calcolo senza confrontare il risultato con una soglia, facendo cioè diretto riferimento al prezzo del nuovo farmaco auto-determinato dall'industria magari assieme ad AIFA (sappiamo che, in Italia, tali prezzi non riflettono una specifica soglia di WTP). Non escludiamo di avere male interpretato qualche passaggio dell'articolo di Ballatori e colleghi, ma a nostro avviso sarebbe invece interessante incorporare nel calcolo una soglia. Su questo punto, inoltre, potrebbe essere interessante un confronto con la precedente letteratura (8-12).

Terzo punto. L'approccio basato sulla densità di probabilità, come descritto da Ballatori e colleghi, è attraente e appare concettualmente superiore rispetto al tradizionale approccio basato su metodi grafici di stima della mediana di OS o PFS. Bisogna però tenere conto che l'approccio tradizionale è sostenuto da tanta letteratura storica [ad esempio, vedasi $(2-10,13)]$, che, andando a ritroso, arriva addirittura fino al secolo scorso $(2,13)$. Comunque, nel caso di utilizzo della OS sa- rebbe interessante eseguire alcuni confronti e vedere se, nelle principali casistiche di trattamento oncologico, la sopravvivenza media e/o la mediana modellizzate secondo la funzione di probabilità, oltre all'indiscusso vantaggio teorico, mostrino rispetto alla mediana grafica - differenze numeriche di un certo rilievo tali da raccomandare l'uso dei parametri modellizzati a fini regolatori.

Last but not least, la controversia su come quantificare il beneficio dei farmaci oncologici sta tornando di prepotente attualità, perché il 22 luglio 2015 l'American Society of Clinical Oncology (ASCO) ha rilasciato un position paper su questa materia (14); in tale documento si propugna una metodologia di analisi che appare molto vicina all'approccio tradizionale sopra citato.

\section{Andrea Messori, Sabrina Trippoli}

HTA, ESTAR (Ente di Supporto Tecnico Amministrativo Regionale) Regione Toscana, Firenze - Italy

\section{Disclosures}

Financial support: No financial support was received for this submission. Conflict of interest: The authors have no conflict of interest.

Indirizzo per la corrispondenza:

Andrea Messori

Responsabile HTA

ESTAR (Ente di Supporto Tecnico

Amministrativo Regionale) - Regione Toscana

50135 Firenze, Italy

andrea.messori.it@gmail.com

\section{Bibliografia}

1. Ballatori E, Ferrante L, Ruggeri B, Roila F. Un nuovo metodo per la determinazione del prezzo equo dei nuovi farmaci oncologici in base al payment-by-results. Global \& Regional Health Technology Assessment. 2015;2(2):97-100.

2. Wright JC, Weinstein MC. Gains in life expectancy from medical interventions-standardizing data on outcomes. N Engl J Med. 1998;339(6):380-6.

3. Fojo T, Grady C. How much is life worth: cetuximab, non-small cell lung cancer, and the $\$ 440$ billion question. J Natl Cancer Inst. 2009;101(15):1044-8.

4. Fadda V, Maratea D, Trippoli S, Messori A. Comparison between real prices and value-based prices of innovative drugs. Part 1 and Part 2. Published 6 December 2010. eBMJ: http:// www.bmj.com/rapid-response/2011/11/03/comparison-between-real-prices-and-value-based-prices-innovative-drugs-0 and http://www.bmj.com/rapid-response/2011/11/03/comparison-between-real-prices-and-value-based-prices-innovative-drugs-par.

5. Messori A, Santarlasci B, Trippoli S, Vaiani M. Controvalore economico del farmaco e beneficio clinico: stato dell'arte della metodologia e applicazione di un algoritmo farmacoeconomico. Pharmacoeconomics-Italian Research Articles. 2003;5: 53-67. http://www.osservatorioinnovazione.net/papers/peccontrovalore.pdf.

6. Messori A, Santarlasci B, Trippoli S. Guadagno di sopravviven- 
za dei nuovi farmaci. Pharmacoeconomics-Italian Research Articles 2004;6:95-104. http://www.osservatorioinnovazione. net/papers/guadagnios.pdf.

7. Messori A, Caccese E, Orsi C, et al. Analisi costo-efficacia: calcolo del guadagno di sopravvivenza e determinazione del costo per anno di vita guadagnato. Bollettino SIFO. 2007;53:86-92. http:// www.osservatorioinnovazione.net/bsifo-messori2007a.pdf.

8. Martone N, Lucioni C, Mazzi S, Fadda V. I nuovi farmaci in oncologia e il loro beneficio in termini di overall survival. Global \& Regional Health Technology Assessment. 2014;1(1):3-15.

9. Martone N, Lucioni C, Mazzi S, Fadda V. Valutazione di costoefficacia dei nuovi farmaci oncologici immessi sul mercato italiano. Global \& Regional Health Technology Assessment 2014;1(2):31-43.

10. Messori A, Trippoli S, Bonacchi M, Sani G. Left-ventricular assist device as destination therapy: application of the payment-byresults approach for the device reimbursement. J Thorac Cardiovasc Surg. 2009;138(2):480-5. http://www.osservatorioinnovazione.net/papers/jtcvs2009.pdf.

11. Messori A, Fadda V, Maratea D, Trippoli S. Differentiating be- tween value-based payment by results and not value-based payment by results. Published 10 November 2010, correction 26 December 2011. eBMJ: http://www.bmj.com/content/341/ bmj.c4388?tab=responses.

12. Messori A, Fadda V, Maratea D, Trippoli S. Un'esigenza di chiarezza: differenziare il payback "value-based" da quello non "value-based". Pharmacoeconomics-Italian Research Articles. 2010;12(3):203-6. http://www.osservatorioinnovazione.net/ papers/pec-it-payback.pdf.

13. Messori A, Becagli P, Trippoli S. Median versus mean lifetime survival in the analysis of survival data. Haematologica. 1997; 82(6):730. http://www.haematologica.org/content/82/6/ 730. long.

14. Schnipper LE, Davidson NE, Wollins DS, et al. American Society of Clinical Oncology Statement: A Conceptual Framework to Assess the Value of Cancer Treatment Options. J Clin Oncol. 2015;33(23)2563-77.

Published online: September 24, 2015 